ISSN 1991-8631

Original Paper

http://indexmedicus.afro.who.int

\title{
Performances de ponte et qualité des œufs des poules pondeuses ISA Brown alimentées avec des rations à base de feuilles séchées de manioc (Manihot esculenta, Crantz)
}

\author{
M. F. HOUNDONOUGBO*, C. A. A. M. CHRYSOSTOME et V. P. HOUNDONOUGBO \\ Laboratoire de Recherche Avicole et de Zoo-Economie, Département de Production Animale, \\ Faculté des Sciences Agronomiques, l'Université d'Abomey-Calavi, 01 BP 526 Cotonou, République du Bénin. \\ *Auteur correspondant; E-mail : fredericmh@gmail.com; Tel. : (+229) 96142965, (+229) 95968136
}

\section{RESUME}

Les performances de ponte et la qualité des œufs de 216 poules pondeuses ISA Brown âgées de 21 semaines ont été déterminées en les alimentant avec des rations contenant des feuilles séchées de manioc (Manihot esculenta, Crantz). Pendant douze semaines, trois groupes de 72 poules chacun (réparties en trois répétitions) sont soumises à trois différentes rations alimentaires (R0, R5 et R10) contenant chacune 0,5 et $10 \%$ de feuilles séchées de manioc. Le taux de ponte des poules est plus élevé avec la ration R0, mais le poids moyen des œufs s'est amélioré avec les rations R5 et R10. L'ingestion alimentaire était similaire dans tous les traitements, mais pendant le premier mois d'essai, l'indice de consommation alimentaire est négativement affecté $(\mathrm{P}<0,05)$ par l'incorporation des feuilles de manioc dans les rations. Une réduction de 12 à 20 FCFA/kg du prix du kilogramme d'aliment par rapport au témoin a été notée mais le coût alimentaire était similaire à partir du deuxième mois. La qualité externe des œufs (indice de forme, épaisseur et poids coquille) était similaire pour toutes les rations. Par contre, les optima de coloration du jaune $(\mathrm{P}<0,05)$ et d'unité Haugh $(\mathrm{P}>0,05)$ sont obtenus avec la ration contenant $5 \%$ de feuilles séchées de manioc. L'incorporation jusqu'à $5 \%$ de feuilles séchées de manioc dans l'alimentation des poules pondeuses a permis d'améliorer les performances de production et la qualité interne des œufs.

(c) 2012 International Formulae Group. All rights reserved.

Mots clés: œufs, poules, incorporation optimal, coût alimentaire.

\section{INTRODUCTION}

Les feuilles des végétaux cultivés ou non sont consommées par les animaux herbivores sous la forme fraîche ou sèche. Certaines feuilles sont utilisées en élevage intensif de volaille en générale, et des poules pondeuses en particulier. En alimentation des poulets, l'incorporation des feuilles de Alchornia cordifolia, (Udedibie and Opara, 1998), de Chronomolaena odorata (Fasuyi et al., 2005), de Leucaena leucocephala (Saparattananan et al., 2005), de Centrosema pubescens (Nworgu et Fasognon, 2007), de Moringa oleifera (Olugbemi et al., 2010), et de Manihot esculenta (Houndonougbo et al., 2012 ; Agunbiade et al., 2007) a été suggérée.

Les feuilles de manioc (Manihot esculenta, Crantz) très disponible dans plusieurs régions d'Afrique sont particulièrement riches en protéines $(21 \%)$ et 
carotènes connu comme étant un colorant du jaune de l'œuf (Ravindran, 1993). Cependant, la présence de l'acide cyanhydrique, du tanin et la pauvreté des feuilles en acide aminés soufrés (méthionine et cystine) limitent leur large utilisation (Ravindran, 1993) en alimentation animale. Le séchage au soleil réduit jusqu'à $90 \%$ le taux de cyanure et de tannin des feuilles de manioc (Eruvbetine et al., 2003). En élevage des poules pondeuses, Saparattananan et al. (2005), ont rapporté une amélioration significative de la production et de la coloration du jaune des œufs comparativement à une ration contenant des feuilles de Leucaena leucocephala et à une ration témoin dépourvue de feuilles.

$\mathrm{Au}$ Bénin, les feuilles de manioc sont largement disponibles après les récoltes et n'ont jusqu'à présent aucune valeur marchande. L'utilisation des feuilles de manioc en alimentation des poules pondeuses, pourrait permettre d'une part, d'améliorer les performances de ponte et la qualité des œufs de poules et d'autre part, de réduire les coûts d'alimentation. Pour ce faire, une expérimentation a été conduite en station sur les poules pondeuses ISA Brown soumises à des rations alimentaires contenant des taux variables de feuilles séchées de manioc doux.

\section{MATERIEL ET METHODES \\ Dispositif expérimental}

L'expérimentation a porté sur 216 poules pondeuses pendant douze semaines entre la $21^{\text {ème }}$ et la $32^{\text {ème }}$ semaine d'âge. Les poules sont logées par paire dans des cages de type californien. Elles sont reparties en 12 groupes de 9 cages chacun. La répartition de ces groupes est faite de façon à éliminer les effets éventuels du vent dominant et de la hauteur des cages sur les performances de ponte et la qualité des œufs. Ainsi, 6 groupes faisaient face au vent dominant et les 6 autres sont orientées contre le vent dominant. A chaque face, 3 groupes sont rangés en hauteur
(115 cm du sol) et 3 groupes sont en bas (70 cm du sol).

Les 9 cages de chaque groupe (18 poules) constituaient une répétition. Chaque traitement alimentaire est distribué à 4 répétitions dont 1 en haut et 1 en bas face au vent dominant, puis 1 en haut et 1 en bas contre le vent dominant.

\section{Aliments et rationnement}

Les rations alimentaires R0, R5 et R10 contenant respectivement 0,5 et $10 \%$ de feuilles séchées de manioc sont distribuées chacun aux poules des 4 groupes/répétitions. La composition centésimale des trois rations testées et leurs valeurs nutritives sont consignées dans le Tableau 2. Ces rations étaient iso-énergétique et iso-protéiniques Tableau 2. Durant l'expérimentation, les poules ont reçu chacune $100 \mathrm{~g}$ d'aliment tel que fabriqué pendant les deux premières semaines de ponte, puis $110 \mathrm{~g}$ de la troisième à la cinquième semaine et $115 \mathrm{~g}$ à partir de la sixième semaine jusqu'à la fin de l'expérimentation. En se basant d'une part, sur le coût de la main d'œuvre pour la récolte et le séchage, et d'autre part, sur les charges de transport, la valeur économique des feuilles séchées de manioc est estimée à $50 \mathrm{FCFA} / \mathrm{kg}$. L'Indice de Consommation alimentaire (IC) a été calculé de deux façons. Ainsi, la quantité d'aliment consommée a été rapportée dans un premier temps à la masse d'œufs produite ( $\mathrm{g}$ aliment/g œuf) et dans un second temps au nombre d'œufs produits ( $g$ aliment/œuf). Le calcul du coût alimentaire a été fait en divisant le coût de l'aliment consommé par nombre d'œufs produits dans la période considérée.

\section{Evaluation de la qualité des œufs}

Les œufs sont collectés par groupe et pesés quotidiennement à 17 heures à l'aide d'un peson électronique de précision $0,1 \mathrm{~g}$. Par semaine, dix-huit (18) œufs du jour sont pris au hasard par répétition pour le texte de qualité, soient 72 œufs par traitement. 
La longueur (L) et le grand diamètre (GD) des œufs sont mesurés à l'aide d'une règle de pied à coulisse pour le calcul de l'Indice de Forme (IF) selon la formule suivante :

$\mathrm{IF}=\frac{\mathrm{GD}}{\mathrm{L}}$

Les œufs pesés sont cassés sur une surface plane. La hauteur de l'albumen et de jaune de l'œuf (vitellus) sont mesurées à l'aide d'un trépied micrométrique électronique de précision 0,01 millimètre. Le poids de l'œuf (W) en gramme et la hauteur de l'albumen $(\mathrm{H})$ en millimètre sont alors utilisés pour calculer l'Unité Haugh grâce à la formule de Haugh (1937):

$\mathrm{HU}=100 \log \left(\mathrm{H}-1,7 \mathrm{~W}^{0,37}+07,57\right)$

La coloration du jaune d'œuf est déterminée en utilisant l'éventail de Roche fait de différents niveaux variant du jaune clair au jaune orange foncé dans l'ordre croissant des chiffres (1 à 15) correspondants à chaque couleur. La pesée de la coquille est réalisée avant et après l'enlèvement de la membrane coquillière suivi du lavage et du séchage. Elle est réalisée à l'aide d'une balance électronique de sensibilité $0,1 \mathrm{~g}$.

L'épaisseur de la coquille est mesurée à l'aide d'un micromètre électronique de précision $0,001 \mathrm{~mm}$ après que les coquilles soient débarrassées de leurs membranes internes. Pour un œuf, trois mesures sont prises au niveau de la région équatoriale de la coquille et une moyenne est calculée.

\section{Analyses statistiques}

Toutes les analyses statistiques ont été réalisées dans le logiciel SAS version 9.1 (2004) par la procédure du General Linear Model (GLM) selon le model suivant :

$\mathrm{Y}_{\mathrm{i}}=\mu+\mathrm{R}_{\mathrm{i}}+\varepsilon_{\mathrm{i}}$

$\mathrm{Y}_{\mathrm{i}}=$ Observation des variables

dépendantes

$\mu=$ Moyenne générale

$\mathrm{R}_{\mathrm{i}}=$ Effet fixe de la ration alimentaire $\varepsilon_{\mathrm{i}}=$ Erreur résiduelle

Les valeurs moyennes des variables et les erreurs standards ont été présentées dans des tableaux avec les probabilités $(\mathrm{P})$ issues de leur comparaison. L'effet de la ration alimentaire est dit significatif si $\mathrm{P}<0,05$.

\section{RESULTATS}

\section{Taux de ponte d'œufs}

Les taux de ponte des poules alimentées avec les rations à base de feuilles séchées de manioc étaient inférieurs à celui obtenu avec la ration témoin R0. Toutefois, entre la $11^{\text {ème }}$ et la $12^{\text {ème }}$ semaine de ponte ( $31^{\text {ème }}$ à $32^{\text {ème }}$ semaine d'âge) la différence s'est considérablement affaiblie (Figure 1). $\mathrm{Au}$ premier mois de ponte, les taux de ponte moyens étaient respectivement de 47,6 ; 42,9 et $53,7 \%$ dans les traitements alimentaires R0, R5 et R10, contre 84,7; 75,4 et $71,7 \%$ au deuxième mois, puis 81,3 ; 71,7 et $74,4 \%$ au troisième mois.

\section{Ingestion alimentaire et indice de consommation alimentaire}

Les poules sont rationnées durant l'expérimentation. Quotidiennement, elles ont ingérées toute la quantité d'aliment servie $(100 \mathrm{~g} / \mathrm{j}, 110 \mathrm{~g} / \mathrm{j}$ et $115 \mathrm{~g} / \mathrm{j}$, respectivement, de la $1^{\text {ère }}$ à la $2^{\text {ème }}$, de la $3^{\text {ème }}$ à la $5^{\text {ème }}$ et à partir de la $6^{\text {ème }}$ semaine).

L'indice de consommation alimentaire était plus élevé chez les poules soumises aux rations $\mathrm{R} 5$ et $\mathrm{R} 10$ à base de feuilles de manioc par rapport à la ration témoin $\mathrm{R} 0$, ceci quelle que soit la méthode de calcul utilisée et l'âge des poules, (Tableau 3). Toutefois, la différence n'était pas significative entre les trois traitements alimentaires pour ce paramètre.

Au cours du premier mois, l'indice de consommation alimentaire (IC) est particulièrement très élevé dans R5 due au faible taux de ponte enregistré à cette période avec cette ration. Pendant le premier mois de ponte, les plus faibles IC sont obtenus avec R0 
et R10. Ceci témoigne d'une bonne entrée en ponte des poulettes nourris avec la ration contenant $10 \%$ de feuilles de manioc. Toutefois, à partir du deuxième mois de ponte, l'indice de consommation était devenu plus faible et stable chez les poules du traitement R0, comparativement aux IC des poules alimentées avec les rations à base de feuilles de manioc.

\section{Prix des aliments pontes et coût alimentaire}

L'incorporation des feuilles séchées de manioc a permis une réduction du prix des aliments R5 et R10 respectivement de 12 et 20 FCFA/kg, par rapport au prix de la ration témoins R0 (Tableau 4). Lorsque le taux d'incorporation des feuilles de manioc séchées passe de 5 à $10 \%$, le prix de l'aliment est réduit de $8 \mathrm{FCFA} / \mathrm{kg}$ sans que les besoins nutritionnels ne soient en deçà des niveaux recommandés en alimentation des poules pondeuses. Par contre, comparativement à la ration témoin, l'incorporation de $5 \%$ de feuilles séchées de manioc dans l'aliment a fait accroître significativement le coût alimentaire en début de ponte, mais pas au cours du deuxième mois de ponte. Cette situation est essentiellement liée à une diminution de la productivité en œufs des poules soumises aux rations R5 et R10 pendant le premier mois d'expérimentation. Ces résultats démontrent que l'utilisation des feuilles de manioc dans l'aliment des poules pondeuses a engendré une baisse significative de l'efficience alimentaire en début de ponte.

\section{Qualité physiques des œufs des poules}

Les informations sur la qualité physique des parties externe et interne des œufs sont présentées par la Figure 2 et le Tableau 5.

Le poids moyen des œufs s'est amélioré durant les trois mois avec l'incorporation des feuilles de manioc dans la ration des poules (Figure 2). Cette amélioration était significative $(\mathrm{P}<0,05)$ au début de la ponte avec une différence moyenne de 5,5 g entre le poids des œufs des poules des rations R5 et R0. Le taux de 5\% de feuilles de manioc dans la ration des poules est apparu comme celui conférant le meilleur poids aux œufs entre la $21^{\text {ème }}$ et $32^{\text {ème }}$ semaine d'âge. Les rations alimentaires ont produit des œufs dont l'indice de forme, l'épaisseur et le poids de la coquille étaient similaires ( $\mathrm{P}$ > 0,05). Par contre, l'étude de la qualité interne des œufs à travers la couleur du jaune, l'épaisseur du jaune et du blanc et l'unité Haugh, a montré des différences significatives $(\mathrm{P}<0,05)$ entre les rations (Tableau 5). Les rations contenant les feuilles séchées de manioc (R5 et R10) ont permis d'améliorer le jaune et le blanc d'œufs qui sont les parties consommables des œufs.

Tableau 1: Dispositif expérimental.

\begin{tabular}{lccc}
\hline Éléments caractéristiques & \multicolumn{3}{c}{ Traitements expérimentaux* } \\
\cline { 2 - 4 } & R0 & R5 & R10 \\
\hline Taux de feuilles de manioc séchées $(\%)$ & 0 & 5 & 10 \\
Poules par traitement & 72 & 72 & 72 \\
Nombre de cage de deux poules & 36 & 36 & 36 \\
Cages en haut face au vent dominant & 9 & 9 & 9 \\
Cages en haut contre le vent dominant & 9 & 9 & 9 \\
Cages en bas face au vent dominant & 9 & 9 & 9 \\
Cages en bas contre le vent dominant & 9 & 9 & 9 \\
\hline
\end{tabular}

*Les traitements expérimentaux sont des rations alimentaires contenant ou non des feuilles de manioc séchées 
Tableau 2: Ingrédients et composition nutritionnelle des aliments expérimentaux des poules en phase ponte.

\begin{tabular}{|c|c|c|c|}
\hline \multirow[b]{2}{*}{ Ingrédients (\%) } & \multicolumn{3}{|c|}{$\begin{array}{c}\text { Aliments Ponte } \\
\text { (A partir de la } 21^{\text {ème }} \text { semaine) }\end{array}$} \\
\hline & R0 & R5 & R10 \\
\hline Feuilles séché de manioc doux & 0 & 5 & 10 \\
\hline Maïs & 55,4 & 50,4 & 49,4 \\
\hline Son de blé & - & 2 & - \\
\hline Tourteau soja & 24 & 24 & 24 \\
\hline Tourteau coton & 8 & 6 & 4 \\
\hline Huile rouge & 1 & 1 & 1 \\
\hline Coquille d'huile & 10 & 10 & 10 \\
\hline Lysine & 0,05 & 0,05 & 0,05 \\
\hline Méthionine & 0,15 & 0,15 & 0,15 \\
\hline Phosphate bi calcique & 0,80 & 0,80 & 0,80 \\
\hline Sel (Nacl) & 0,30 & 0,30 & 0,30 \\
\hline Prémix $^{1}(\mathrm{CMV})$ & 0,25 & 0,25 & 0,25 \\
\hline Sulfate de fer & 0,03 & 0,03 & 0,03 \\
\hline Total & 100 & 100 & 100 \\
\hline \multicolumn{4}{|l|}{ Composition nutritionnelle } \\
\hline Matière Sèche (\%) & 88,6 & 85,5 & 82,4 \\
\hline Cellulose brute $(\%)$ & 4,16 & 4,43 & 4,52 \\
\hline Energie Métabolisable (kcal/kg MS) & 2664 & 2629 & 2668 \\
\hline Protéine brute $(\%)$ & 18,6 & 18,7 & 18,7 \\
\hline Lysine $(\%)$ & 0,97 & 0,92 & 0,87 \\
\hline Méthionine $(\%)$ & 0,46 & 0,43 & 0,41 \\
\hline Acide aminé soufré (\%) & 0,78 & 0,74 & 0,70 \\
\hline Calcium $(\%)$ & 4,05 & 4,16 & 4,25 \\
\hline Phosphore total (\%) & 0,57 & 0,64 & 0,69 \\
\hline
\end{tabular}

Tableau 3: Indice de consommation (IC) alimentaire (g aliment/g œuf et $g$ aliment/œuf) des poules nourries avec des rations à base de feuilles séchées de manioc doux.

\begin{tabular}{|c|c|c|c|c|c|}
\hline Mois de ponte & R0 & $\mathbf{R 5}$ & R10 & $\mathbf{E S}$ & Probabilité \\
\hline \multicolumn{6}{|c|}{ Indice de consommation ( $\mathrm{g}$ aliment /g œuf) } \\
\hline 1 & $5,7^{\mathrm{a}}$ & $9,5^{\mathrm{b}}$ & $5,8^{\mathrm{a}}$ & 0,33 & 0,02 \\
\hline 2 & 2,4 & 2,7 & 2,9 & 0,17 & 0,70 \\
\hline 3 & 2,4 & 2,9 & 2,8 & 0,15 & 0,65 \\
\hline \multicolumn{6}{|c|}{ Indice de consommation (g aliment /œuf) } \\
\hline 1 & $239^{\mathrm{a}}$ & $463^{\mathrm{b}}$ & $271^{\mathrm{a}}$ & 10,5 & 0,02 \\
\hline 2 & 134 & 151 & 159 & 5,27 & 0,61 \\
\hline 3 & 134 & 165 & 157 & 6,01 & 0,42 \\
\hline
\end{tabular}


Tableau 4: Prix des aliments formulés (FCFA/kg aliment) et coût alimentaire (FCFA/œuf) chez les poules pondeuses ISA Brown.

\begin{tabular}{lcccccc}
\hline & Mois de ponte & R0 & R5 & R10 & ES & Probabilité \\
\hline Prix des aliments & - & 207 & 195 & 187 & - & - \\
\hline Coût alimentaire & 1 & $49^{\mathrm{a}}$ & $90^{\mathrm{b}}$ & $51^{\mathrm{a}}$ & 2,0 & 0,003 \\
& 2 & 28 & 29 & 30 & 1,6 & 0,750 \\
& 3 & 28 & 32 & 29 & 1,5 & 0,560 \\
\hline
\end{tabular}

${ }^{\mathrm{a}, \mathrm{b}} \mathrm{Les}$ moyennes suivies de différentes lettres sur la même ligne sont significativement différents $(\mathrm{P}<0,05)$

Tableau 5: Carractéristiques physiques des parties externe et interne des œufs des poules alimentées avec des rations à base de feuilles de manioc séchées.

\begin{tabular}{|c|c|c|c|c|c|c|c|}
\hline \multirow[t]{2}{*}{$\begin{array}{l}\text { Rations } \\
\text { alimentaires }\end{array}$} & \multicolumn{7}{|c|}{ Qualités physiques des oeufs } \\
\hline & $\begin{array}{l}\text { Indice } \\
\text { forme }\end{array}$ & $\begin{array}{l}\text { Couleur } \\
\text { jaune }\end{array}$ & $\begin{array}{c}\text { Épaisseur } \\
\text { jaune } \\
(\mathrm{mm})\end{array}$ & $\begin{array}{c}\text { Épaisseur } \\
\text { albumen } \\
(\mathrm{mm})\end{array}$ & $\begin{array}{c}\text { Unité } \\
\text { Haugh }\end{array}$ & $\begin{array}{c}\text { Poids } \\
\text { coquille } \\
(\mathrm{g})\end{array}$ & $\begin{array}{c}\text { Épaisseur } \\
\text { coquille } \\
(\mathbf{m m})\end{array}$ \\
\hline R0 & 77,8 & $2,00^{\mathrm{a}}$ & 17,5 & 7,57 & 85,1 & 7,52 & 0,37 \\
\hline $\mathrm{R} 5$ & 78,3 & $5,41^{\mathrm{b}}$ & 18,1 & 8,20 & 90,5 & 7,66 & 0,39 \\
\hline $\mathrm{R} 10$ & 78,1 & $4,53^{\mathrm{b}}$ & 18,1 & 8,07 & 89,0 & 7,60 & 0,39 \\
\hline $\mathrm{ES}$ & 1,81 & 0,20 & 0,97 & 0,54 & 2,10 & 0,49 & 0.01 \\
\hline Probabilité & 0,68 & 0.02 & 0,04 & 0,03 & 0,02 & 0,82 & 0,67 \\
\hline
\end{tabular}

${ }^{a, b}$ Les moyennes suivies de différentes lettres sur la même ligne sont significativement différents $(\mathrm{P}<0,05)$

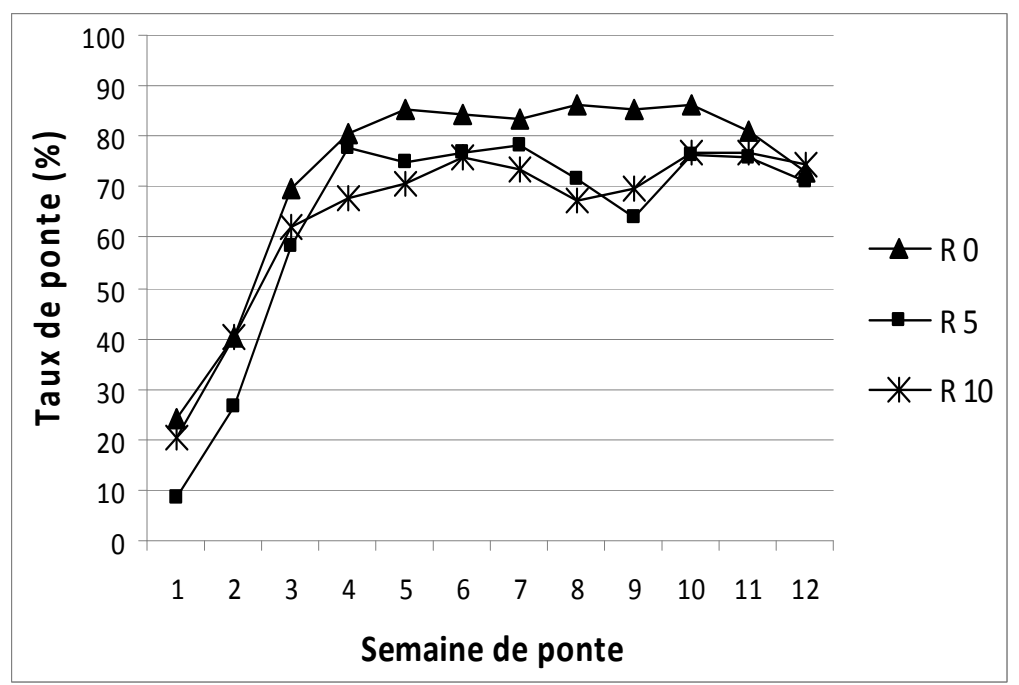

Figure 1: Courbe de taux de ponte des poules. R0 = Ration dépourvue de feuille de manioc; $\mathrm{R} 5=$ Ration contenant $5 \%$ de feuille de manioc ; R10 = Ration contenant $10 \%$ de feuille de manioc. 


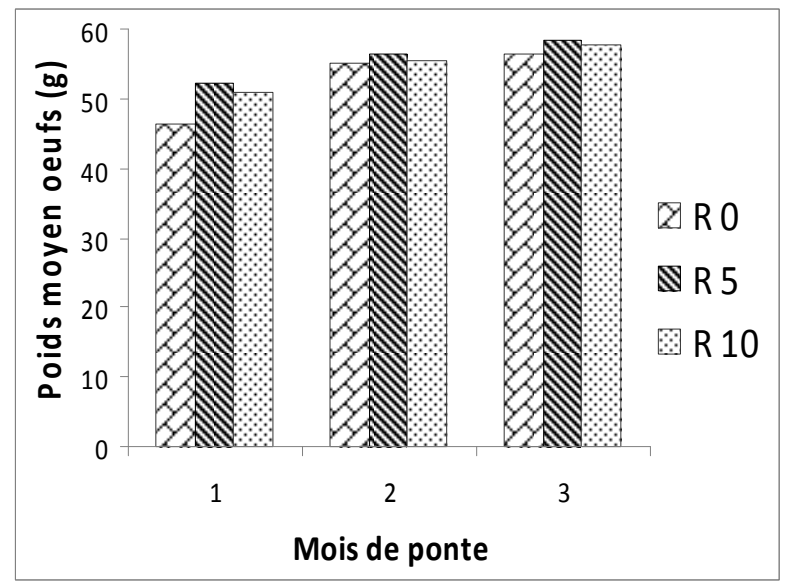

Figure 2: Poids moyen des œufs. R0 = Ration dépourvue de feuille de manioc; R5 = Ration contenant $5 \%$ de feuille de manioc $; \mathrm{R} 10=$ Ration contenant $10 \%$ de feuille de manioc.

\section{DISCUSSION}

\section{Taux de ponte d'œufs}

Il ressort de la présente étude que l'incorporation des feuilles séchées de manioc dans la ration alimentaire des poules pondeuses a eu une influence négative sur leur taux de ponte. Ceci peut être dû aux effets des facteurs antinutritionnels (acide cyanhydrique et tanin) et de la cellulose brute qui sont dans les feuilles séchées de manioc des rations R5 et $\mathrm{R} 10$. Des résultats similaires sur le taux de ponte des poules pondeuses White leghorn (de la même tranche d'âge) sont rapportés par Kakengi et al. (2007) avec l'utilisation des feuilles de Moringa oleifera dans l'alimentation de poulettes âgées de 21 semaines.

\section{Ingestion alimentaire et indice de consommation}

La protéine et l'énergie métabolisable contenues dans chacune des trois rations expérimentales étaient presque similaires. De plus, le taux de cellulose brute est maintenu à une valeur inférieure à $5 \%$ dans toutes les rations malgré sa légère augmentation (6.5 et $8.7 \%$ ) dans les rations à feuilles de manioc (R5 et R10 respectivement). De légères baisses du taux de matière sèche sont par contre notées avec l'introduction des feuilles séchées de manioc dans les rations alimentaires des poules. Toutefois, l'incorporation des feuilles séchées de manioc dans les rations a entrainé de légères réductions des taux de lysine, méthionine et acide aminé soufré, confirmant ainsi leur pauvreté en acides aminés (Ravindran, 1993 ; Agunbiade et al., 2002 et Houndonougbo et al., 2012). Par contre, il en a découlée une amélioration des taux de calcium et de phosphore total des rations alimentaires R5 et R10 contenant des feuilles séchées de manioc.

La similarité d'ingestion alimentaire observée chez les poules alimentées avec les trois rations indique une bonne ingestion de celles-ci. Ce résultat contredit ceux obtenus par plusieurs auteurs chez des poules pondeuses, poulettes et poulets de chair. Nworgu et Fasogbon (2007) ont obtenu des différences significatives d'ingestion moyenne d'aliment en utilisant les feuilles de Centrosema pubescens comme un supplément protéique dans la ration de poulettes Black Nera âgées de 10 semaines. Une augmentation significative et progressive des ingestions d'aliment et de matière sèche est rapportée par Kakengi et al. (2007) chez des poulettes White Leghorn de 21 semaines d'âge nourries pendant 13 semaines avec des rations contenant des taux de 10 et $20 \%$ de feuilles de Moringa oleifera. La substitution de la farine de poisson par un concentré protéique extrait 
des feuilles de manioc dans l'alimentation des poulets de chair a induit des différences significatives d'ingestion d'aliment (Fasuyi et Aletor, 2005). Par contre, une similarité d'ingestion d'aliment est rapportée chez des poulettes ISA Brown (Houndonougbo et al., 2012) et des poules pondeuses Isa Brown and Nera Black à la $50^{\text {ème }}$ semaine de ponte (Agunbiade et al., 2007) soumise à des rations à base de feuilles de manioc.

Les poules soumises aux rations contenant des feuilles séchées de manioc (R5 et R10) présentaient des indices de consommation alimentaire plus élevés par rapport à celui des poules alimentées avec la ration témoin $\mathrm{R} 0$ dépourvue de feuilles de manioc. Au cours du premier mois, l'IC des poules alimentées avec la ration R5 était significativement $(\mathrm{P}<0.05)$ le plus élevé. Par contre, pendant les deux mois suivant, les différences d'IC n'étaient plus significatives $(\mathrm{P}>0.05)$. Les valeurs moyennes d'IC (3,5 à $5,03 \mathrm{~g}$ aliment/g œuf) obtenues dans la présente étude sont plus élevées que celles indiquées par Kakengi et al. (2007) avec l'utilisation des feuilles de Moringa oleifera dans l'alimentation de poulettes White leghorn âgées de 21 semaines (2,64 à 3,29 g aliment/g œuf), et par Mutahar et al (2011) avec les feuilles de luzerne chez des poules White Hisex entre 20 et 28 semaines d'âge (2,01 à 3,02 g aliment/g œuf).

\section{Prix des aliments ponte et coût alimentaire}

L'incorporation des feuilles séchées de manioc dans l'alimentation des poules pondeuses a permis de réduire d'environ 5 à 9\% le prix d'un kilogramme d'aliment.

La baisse de l'efficience alimentaire en début de ponte serait liée au taux élevé de cellulose $(11,4 \%)$ des feuilles de manioc, ce qui réduit à cet âge leur valorisation par la volaille à haute productivité. Toutefois, cette pratique alimentaire permet de valoriser en alimentation animale les feuilles de manioc qui sont des ressources alimentaires peu valorisées lors de l'exploitation en masse des racines de manioc.

\section{Qualités des œufs des poules}

L'introduction de feuilles séchées de manioc dans la ration alimentaire des poules a influencé le poids moyen des œufs à des degrés différents. Les œufs avec les poids les plus élevés sont obtenus chez les poules alimentées avec la ration contenant 5\% de feuilles de manioc; mais le poids des œufs chute légèrement à $10 \%$ d'incorporation de feuilles de manioc dans l'aliment. Kakengi et al (2007) sont parviennus à des résultats similaires avec la substitution des graines de tournesol par des feuilles de Moringa oleifera dans l'alimentation de poulettes âgées de 21 semaines. En effet, au taux de substitution de $5 \%$, ces auteurs ont obtenu des œufs avec les poids les plus élevés; mais une réduction du poids des œufs est notée avec l'augmentation du taux de feuilles de Moringa oleifera. Par contre, Bhatnagar et al. (1996) n'ont pas enregistré de différence significative entre les rations contenant différents niveaux $(0,5$ et $10 \%$ ) de feuilles de Leuceana leucocephala en termes de poids des œufs de poules. Mais ces auteurs ont obtenu des chutes de poids des œufs avec un taux d'incorporation de $20 \%$ de Leuceana leucocephala dans la ration. Par ailleurs, Saparattananan et al. (2005) sont parvenus à la même conclusion sur le poids moyen des œufs avec des rations contenant des feuilles de manioc ou de Leucaena leucocephala.

L'incorporation de feuilles séchées de manioc jusqu'à un taux de $10 \%$ dans la ration alimentaire des poules pondeuses n'a pas eu d'effet significatif $(\mathrm{P}>0,05)$ sur l'indice de forme des œufs, l'épaisseur et le poids de la coquille des œufs. Ces résultats confirment ceux de plusieurs auteurs. En effet, l'incorporation de feuilles de Chromolaena odorata $(2,5$ à $7,5 \%)$ dans la ration alimentaire de poules pondeuses à partir du huitième mois de ponte, est restée sans effet significatif sur le poids moyen des œufs et l'épaisseur de leur coquille (Fasuyi et al., 2005). Saparattananan et al. (2005) sont parvenus à la même conclusion sur l'épaisseur de la coquille des œufs avec des rations 
contenant des feuilles de manioc ou de Leucaena leucocephala. Néanmoins, Mutahar et al. (2011) ont rapporté des effets significatifs de l'utilisation des feuilles de luzerne dans l'alimentation des poules pondeuses sur le poids moyen des œufs, l'épaisseur de la coquille et l'indice de forme des œufs.

Il ressort de l'analyse de la qualité interne des œufs que les hauteurs de vitellus et d'albumen ainsi que la couleur du jaune d'œuf et l'unité Haugh se sont significativement améliorées suite à l'incorporation des feuilles séchées de manioc dans la ration alimentaire des poules. De plus le taux de $5 \%$ de feuilles séchées de manioc a permis d'optimiser la qualité interne des œufs. Les différences significatives observées dans la coloration du jaune d'œufs pourraient se justifier par la présence dans les feuilles de manioc de caroténoïdes (Ravindran, 1993) qui sont métabolisées dans l'intestin des poules et sont ainsi converties en vitamine A (Onwudike et Oke (1978) et Fasuyi et al. (2005). Ces résultats sont similaires à ceux de Fasuyi et al. (2005) qui rapportent de significatifs effets de l'utilisation des feuilles de Chromolaena odorata dans la ration des poules pondeuses sur la coloration du jaune de l'œuf. Par contre, les feuilles de Chromolaena odorata sont restées sans effet sur l'unité Haugh et la hauteur d'albumen. L'incorporation de feuilles séchées de manioc jusqu'a $5 \%$ dans l'alimentation des poules pondeuses permet d'améliorer les performances de production et la qualité interne des œufs.

\section{REFERENCES}

Agunbiade JA, Adeyemi OA, Depoju OA, Lawal OA. 2002. The use of whole cassava meal and leaf meal in broiler diets. Tropical Journal of Animal Sciences, 5: 161-173.

Agunbiade JA, Adeyemi OA, Ashiru OM, Awojobi HA, Taiwo AA, Oke DB, Adekunmisi AA. 2007. Replacement of Fish Meal with Maggot Meal in Cassava- based Layers' Diets. The Journal of Poultry Science, 44: 278-282.

Bhatnagar R, Kataria M, Verna SVS. 1996. Effect of dietary Leucaena leaf meal on the performance and egg characteristics in white leghorn hens. Indian Journal of Animal Science, 66(12): 1291-1294.

Evuvbetine D, Tajudeen ID, Adeosun AT, Olojedde AA. 2003. Cassava (Manihot esculenta, Crantz) leaf and tuber concentrate in diet for broiler chickens. Bioresources Technology, 86: 277-281.

Fasuyi AO, Aletor VA. 2005. Protein Replacement Value of Cassava (Manihot esculenta, Crantz) Leaf Protein Concentrate (CLPC) in Broiler Starter: Effect on Performance, Muscle Growth, Haematology and Serum Metabolites. International Journal of Poultry Science, 4(5): 339-349.

Fasuyi AO, Fajemilehin SOK, Omojola AB. 2005. The Egg Quality Characteristics of Layers Fed Varying Dietary Inclusions of Siam Weed (Chromolaena odorata) Leaf Meal (SWLM). International Journal of Poultry Science, 4(10): 752-757.

Haugh RR, 1937. The Haugh unit for measuring egg quality. US Egg Poult. Mag., 43: 552-555.

Houndonougbo MF, Chrysostome CAAM, Houndonougbo VP. 2012. Performances bioeconomiques des poulettes alimentees avec des rations a base de feuilles sechees de manioc (Manihot esculenta). International Journal of Biological and Chemical Sciences, 6(2): 670-676.

Kakengi AMV, Kaijage JT, Sarwatt SV, Mutayoba SK, Shem MN, Fujihara T. 2007. Effect of Moringa oleifera leaf meal as a substitute for sunflower seed meal on performance of laying hens in Tanzania. Livestock Research for Rural Development, 19(8). Online.

Mutahar AA, Mohamed ES, Talha EA. 2011. Effects of dietary inclusion of different levels of alfalfa leaf meal on laying hens' performance and egg quality. Research 
Opinions in Animal and Veterinary Sciences, 1(11): 748-753.

Nworgu FC, Fasogbon FO. 2007. Centrosema (Centrosema pubescens) Leaf Meal as Protein Supplement for Pullet Chicks and Growing Pullets. International Journal of Poultry Science, 6(4): 255-260.

Olugbemi TS, Mutayoba SK, Lekule FP. 2010. Evaluation of Moringa oleifera leaf meal inclusion in cassava chip based diets fed to laying birds. Livestock Research for Rural Development, 22(6). Online.

Onwudike OC, Oke OL. 1978. Total substitution of leaf protein in the ration of laying hens. Poultry Science, 65: 12011204.

Ravindran V. 1993. Cassava leaves as animal feed: Potential and limitations. Journal of the Science of Food and Agriculture, 61(2): 141-150.
Saparattananan W, Kanto U, Juttupornpong S, Engkagul A. 2005. Utilization of cassava meal and cassava leaf in layer diets on egg quality and protein content in egg. Proceedings of 43rd Kasetsart University Annual Conference, Thailand, 1-4 February, 2005. Subject: Animals 2005 pp. 43-52.

Statistic Analysis System (SAS). 2004. User's Guide Statistic: Analysis System Procedure Version 9.1.2. SAS Institute Inc. Cary, NC: USA.

Udedibie ABI, and Opara CC. 1998. Responses of growing broilers and laying hens to the dietary inclusion of leaf meal from Alchornia cordifolia. Animal Feed Science and Technology, 71: 157-164. 